\author{
A. Tamadon *, D. J. Pons, D. Clucas \\ University of Canterbury, Department of Mechanical Engineering, 8140 Christchurch, \\ New Zealand \\ *abbas.tamadon@pg.canterbury.ac.nz
}

\title{
ANALOGUE MODELLING OF FLOW PATTERNS IN BOBBIN FRICTION STIR WELDING BY THE DARK-FIELD/BRIGHT-FIELD ILLUMINATION METHOD
}

\begin{abstract}
The flow-inducing effect of the bobbin-tool features (tri-flat pin and scrolled shoulder) were replicated by a simple analogue model for aluminium welds by layered plasticine samples. Flow patterns of the weld zone were clarified by a typical stereomicroscopy instrument assisted by dark-field/bright-field illumination. The effects of the pin features, specifically threads and flats in centre of bond zone and scrolled shoulder in sides of stirred zone, were identified. This study shows that internal flow features for BFSW welds is transferable from the friction stir welding process to the functional metal forming processes such where the shearing can extensively affect the microstructure. The similarity between the flow pattern of the provided aluminium samples and the plasticine analogue can validate the accuracy of the flow model presented in this work.
\end{abstract}

Keywords: FSW; bobbin-tool; analogue modelling; flow patterns; darkfield/brightfield

\section{INTRODUCTION}

Friction stir welding (FSW) is a process whereby the weld structure is formed by a plastic deformation involving a rotating non-consuming tool being driven through two pieces of substrate material [1,2]. The general area of interest is welding aluminium sheet, and there are many applications for this in marine, automotive, and aerospace fabrication [3,4]. Weld properties are as good as or better than those from conventional thermal fusion processes, but making a reliable weld is challenging.

During the FSW process, two metal pieces are welded by entering a non-consumable rotating pin into the interface of the plates [5-7]. By a circumferential contact of the supportive shoulder with the surface of the plates, the rotating pin is inserted into the joint interface whereby an appropriate engagement with plates begins the plastic deformation at the touching edges [8-10].

By the advancing movement of the rotating tool through the joint interface, the softened and heated material of workpiece plates flows around the pin [11-13]. The plasticized mass at 
the leading edge of the rotating tool transports to the trailing edge of the tool, where consolidates in form of the solid-phase weld [14-17]. The magnitude of the longitudinal and rotational speeds has a key role in the formation and quality of the stirring zone around the tool. Regarding the stirring zone, the side where the longitudinal and rotational speeds are in the same direction is called the advancing side (AS), and the opposite side of the stirring zone where the longitudinal and rotational speeds have different directions is called the retreating side (RS) $[18,19]$. The stirring action does not melt the workpiece material to be welded but yields it to a plastic state which occurs in temperatures much below the melting point (Tm) [20-22]. Hence, the solidification defects are absent in FSW welds, compared to the fusion welding processes. The schematic of friction-stir welding is shown in Fig. 1.

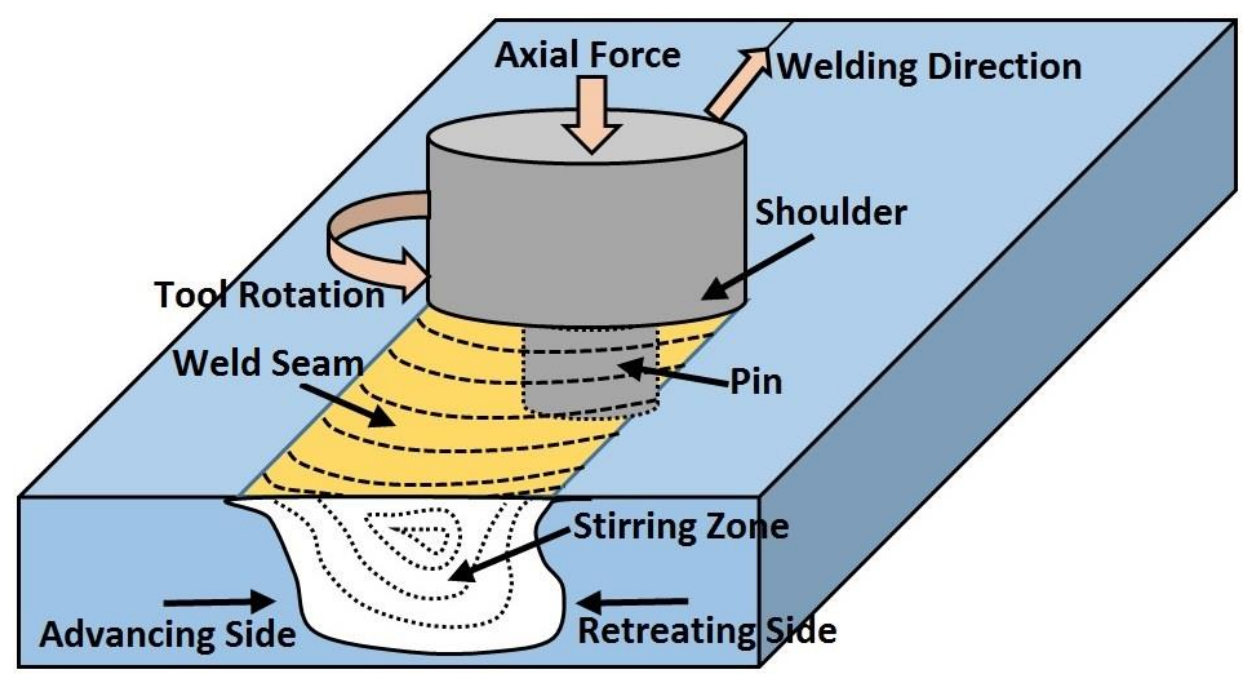

Fig. 1. Schematic of the Friction Stir Welding process

The geometry of the tool can extremely influence the generation of the frictional heat and the subsequent plastic flow at the location of the stirring zone. To control these variables, different innovative designs have been developed to obtain the most effective tool for the optimum stirring action during the welding process.

Bobbin-tool is an innovative tool variant of the conventional-FSW process, whereby a self-reacting double-shoulder configuration connected by a centred-pin ploughing along the butt-interface of the workpiece plates [23], with no backing anvil and the axial force during the process [3]. The elevated dynamic interaction of the tool-workpiece creates much more friction condition at the greater contact interface of the double opposing shoulders and the fully-penetrated pin, compared to the conventional-FSW [4]. Therefore, the plastic yielding and stirring at the bonding track can be attained at lower welding speeds, rotation speed $(\omega)$ and advance speed (V). While the tool advances along the weld line (Fig. 2), the mutual interaction of the speeds $(\omega, \mathrm{V})$ displaces the softened mass from the leading edge of the rotating tool to be deposited at trailing edge of the tool [24]. These differences lead to different flow regimes, where the inconsistency between the process variables can cause more severe failures within the weld structure, different to conventional-FSW (see Fig. 2). 


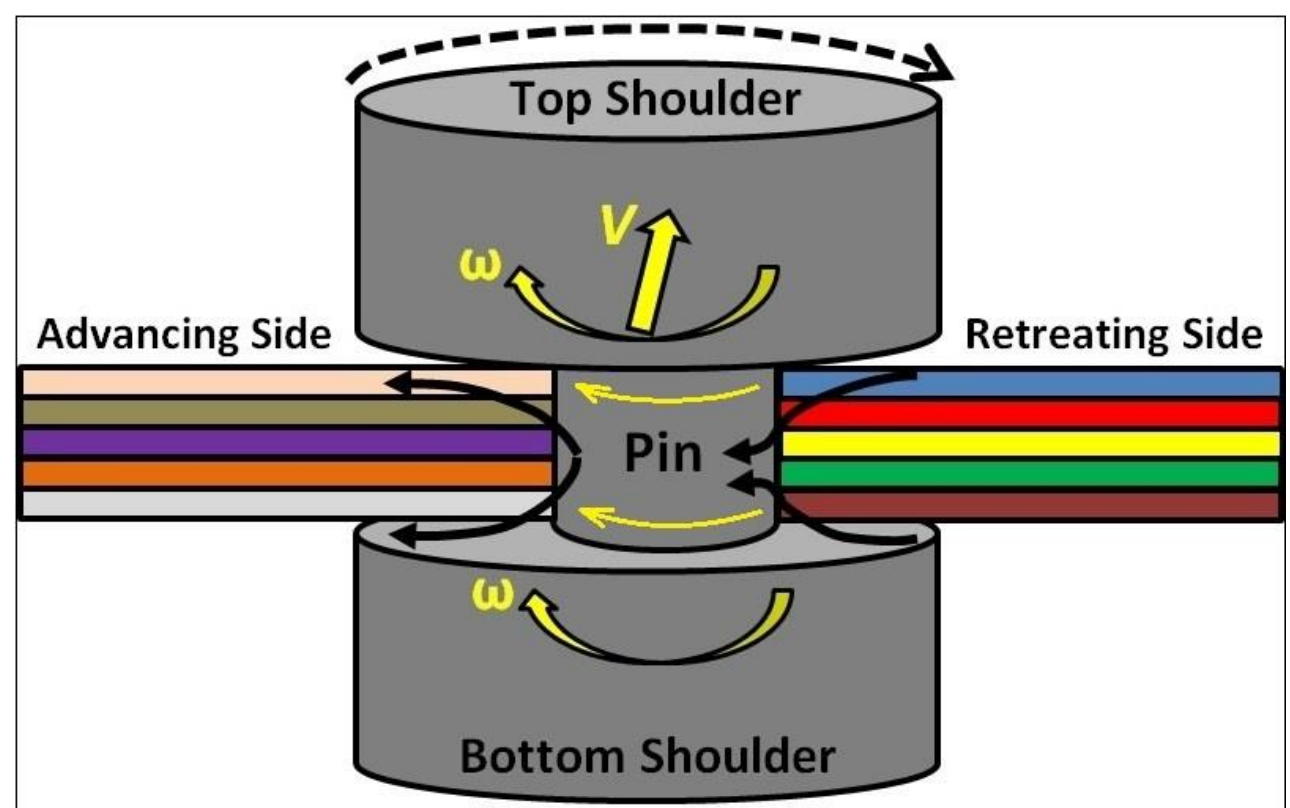

Fig. 2. Schematic of the Bobbin Friction Stir Welding process for a butt-joint position; flow interaction between the bobbin-tool and the plasticine workpiece

One of the main issues for the study of the FSW process is to find an effective method for the understanding of the internal material flows. To study the actual flow regimes of the weld structure, the details of the flow features need to be visualised within the cross-section [25]. This is intrinsically challenging for aluminium welds to reveal the material flow paths, because of insufficient response to conventional metallographic reagents [18].

The work of Sued et al [17] provided new insights into the flow mechanisms and how the failure of the flows caused weld defects. However, the nature of the process makes it difficult to freeze the actual flow and this makes it difficult to validate the computational model. A solution to this, and the subject of the present research, is to use an analogue model for the study of the flow mechanism during the bobbin-FSW process. The aim is to create a plasticine slab, comprising multiple layers of distinct colours. This provides a means to cut crosssections afterwards and identify where the colours have been displaced in the stirred zone of FSW weld. A benefit of this approach is to develop the elucidation of the internal flow regimes, in a physical model of the weld zone. By identifying the causality between flow regimes and visualizing of the plastic deformation features, a flow-based analogue model can explain the origins of the physical defects

The research question is to discover how to perform analogue modelling of FSW using colour layers and grids of plasticine, and thus apply this to determine the flow inside the weld. The area under examination is bobbin friction stir welding (BFSW). We conduct the actual bobbin-tool FSW process on the plasticine slabs instead of aluminium plates. The microstructure of weld will be studied by using a typical stereomicroscope using darkfield/bright-field illumination to find a further explanation for flow regimes within the BFSW weld structure. 


\section{EXPERIMENTAL}

The analogue test of the bobbin friction stir welding was modelled with multi-layered plasticine slabs (New Clay Products, Gordon Harris Ltd., London, UK). By using different colours of plasticine it is easy to distinguish the material flow trajectories. Hence, different colours of plasticine blocks were rolled to a thickness of $2 \mathrm{~mm}$, stacked on top of each other to form the $10 \mathrm{~mm}$ thick slab. The plasticine blocks were subject to heat treatment to improve the adhesiveness between the layers. The blocks were heated to a temperature of $60^{\circ} \mathrm{C}$ for a period of two hours. The heated blocks of plasticine were cooled gradually to $-4^{\circ} \mathrm{C}$ as the working temperature. To validate the analogue plasticine model, the 6082-T6 aluminium plates also were processed by similar bobbin-tool.

The welding test was performed by a single piece bobbin-tool that consists of a topshoulder, a bottom-shoulder and a threaded tri-flat pin in the middle, (see Fig. 3). The bobbintool was made of 3D-printed VeroClearTM plastic material, manufactured by (Stratasys Connex 3D Printer, Eden Prairie, MN, USA). The plastic-made tool has a better consistency with the non-metallic plasticine samples, where the flow feature details need to be replicated on the multi-layered plasticine analogue. The aluminium plates were processed by a bobbintool made of $\mathrm{H} 13$ tool steel, with similar geometric features (threads, flats, scrolls). In both of aluminium and plasticine trials, the pin tool was threaded right-to-left to provide an upward flow pumping during clockwise rotation.
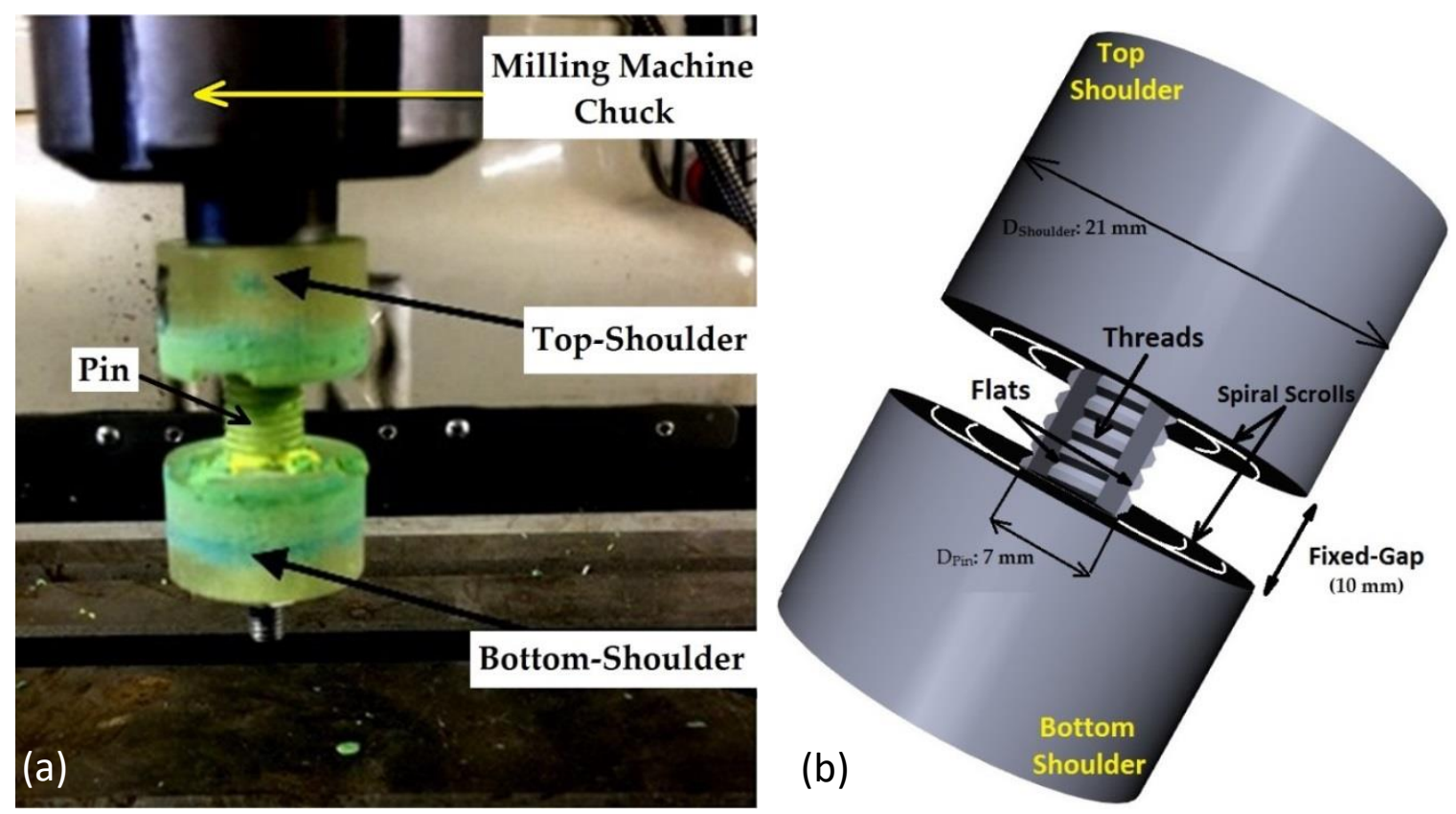

Fig. 3. Schematic of the BFSW process and the fully-features bobbin tool; (a) 3D-printed bobbin-tool after interaction with the workpiece, utilised for the Analogue modelling of the BFSW, installed on a manual milling machine; (b) the 3D model of the bobbin-tool utilised in welding tests, a fixed-gap fully-featured tool with a tri-flat centred pin, connected to the top and bottom shoulders with spiral scrolled surface

Because of the limitation of the material instability under the stirring processing condition, the welding speeds (spindle rotational speeds; $\omega$ and longitudinal welding speed; $\mathrm{V})$, were selected in the minimum range speeds of the milling machine, just sufficient for the establishment of a bonded weld. In this regard, the plasticine analogue samples were 
processed under a constant longitudinal welding speed ( $\mathrm{V}=50 \mathrm{~mm} / \mathrm{min}$ ) with four different spindle rotational speeds $(\omega=50,60,75,100 \mathrm{RPMs})$, just enough to achieve a uniform defect-free weld structure. In case of aluminium plates, the welding speed set of $(\mathrm{V}=300$ $\mathrm{mm} / \mathrm{min})$ and $(\omega=400,500,600 \mathrm{RPMs})$ was applied to produce different flow patterns within the weld structure.

The trials were run using a CNC milling machine (OKUMA, MX-45VAE Model, Oguchi Plant, Aichi, Japan), in a clockwise tool rotation when viewed from above. It means that the AS is on the true-left of the locus of the weld-line and the RS is on the true-right (both at the top-view and the cross-section). The tool performance in a clockwise rotating can centralize the stirring mass inwards the weld position, hence avoid from the material loss or mass ejection in the form of flash defect.

The butted plasticine slabs, with dimensions of $250 \mathrm{~mm}$ (length) $\times 75 \mathrm{~mm}$ (width) $\times 6$ $\mathrm{mm}$ (thickness), were rigidly fixed between clamp bars to ensure that no lateral movement during the welding process occurred. To improve the stability of the layered plasticine during the process, glycerine liquid was used as the lubricant between the bobbin-tool and substrate. Severe shearing flow underneath the shoulder, sub-shoulder scrolled patterns can affect the overflow streams of the soft plasticine. Also clogging of the mass flow can occur between the surface scroll features of the tool and the workpiece. The amount of lubricant was optimized not to affect the frictional situation of the process. It was also enough to prevent any uncontrolled mass digging and clogging between should/pin and workpiece.

The aluminium welding trials also were conducted on the plates with the dimensions similar to the plasticine samples. More technical details of the BFSW processing of the analogue plasticine and aluminium plates are listed in Table 1.

The flow patterns of the BFSW weld region were studied at the cross-section of the analogue plasticines by a dual-power stereomicroscope (Olympus Metallurgical Microscope, Tokyo, Japan), facilitated by a dark-field/bright-field objectives exposure. The aluminium weld cross-sections were prepared by a standard set of metallographic polishing to obtain a mirror level surface. The samples were then etched by the Keller's reagent and were studied by the same microscopy procedure.

Table 1. The operational parameters of the BFSW process for the plasticine analogue and aluminium plates

\begin{tabular}{cc}
\hline Welding Parameters & amount \\
\hline D $_{\text {Shoulder }}(\mathrm{mm})$ & 21 \\
D $_{\text {Pin }}(\mathrm{mm})$ & 7 \\
$D_{\text {Shoulder }} / \mathrm{D}_{\text {Pin }}$ & 3 \\
Workpiece Thickness $(\mathrm{mm})$ & 10 \\
Tool's Biting Gap $(\mathrm{mm})$ & 10 \\
Spindle rotational speed, $\omega(\mathrm{rmm})$, for plasticine & $50,60,75,100$ \\
longitudinal welding speed, $V(\mathrm{~mm} / \mathrm{min})$, for plasticine & 50 \\
Spindle rotational speed, $\omega(\mathrm{rpm})$, for aluminium & $400,500,600$ \\
longitudinal welding speed, $V(\mathrm{~mm} / \mathrm{min})$, for aluminium & 300 \\
Thread Pitch $(\mathrm{mm})$ & 1.25 \\
Number of Threads & 8 \\
Working Temperature $\left({ }^{\circ} \mathrm{C}\right)$ & -4 \\
\hline
\end{tabular}




\section{RESULTS AND DISCUSSION}

After the welding process, the samples were cooled in room temperature and then were cut with a wire cutter, perpendicular to the welding direction. For microscopic analysis, the cross sections were studied using a stereomicroscope. To clarify voids and cracks inside the structure, the dark-field/bright-field Illumination method was used. Using this technique a variety of flow patterns in different zones of weld were extracted. As the tool rotation was clockwise, in all cross-sections the advancing side (AS) of the weld is situated on the left side of the weld region, and the retreating side (RS) is located on the right.

Fig. 4 shows the position of the selected region for the microscopic observation from the analogue plasticine sample. According to Fig. 4, the samples in Figures 5-8 were selected from the stirring zone in the area between the position of the shoulder and pin, near the advancing side border, where the shearing effect of the tool components (pin and shoulder) is maximum. Therefore the flow patterns can show a better effect of the shearing plastic induced by the tool.

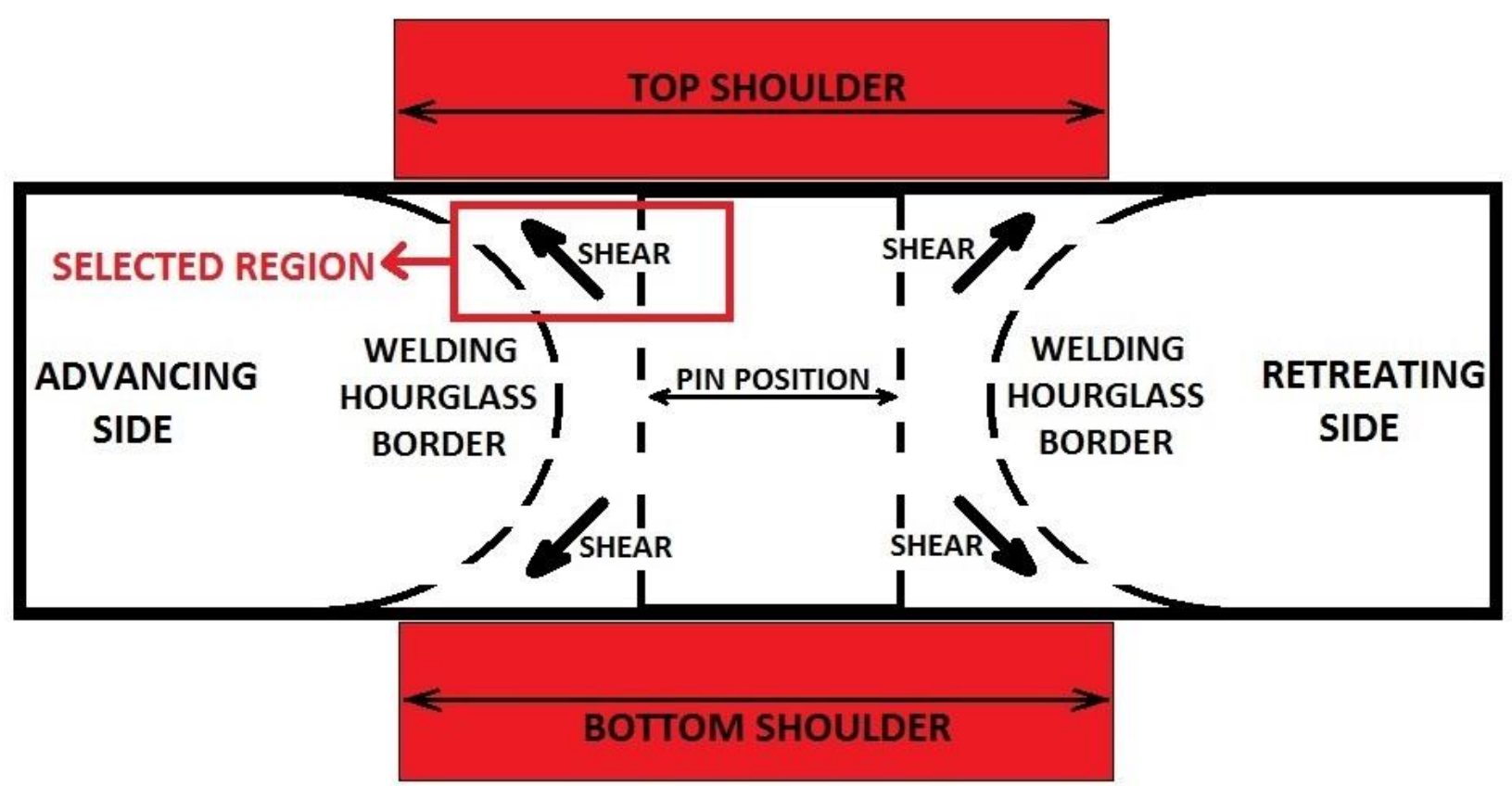

Fig. 4. The schematic of the weld structure; the red-colour rectangular frame shows the selected region of the stirring zone for the microscopic studies in Figures 5-8

Fig. 5. illustrates the stress concentration patterns as a result of the interaction of the shoulder and pin with the material. Because of higher stresses caused by the shoulder surface, branches are stretched from centre of stirred zone to the sub-surface area.

As the stress rates convert to a continuous mode, the focus points in the structure connect together to create plane strains. The pattern of these plain strain regimes is observed through Fig. 6.

Due to the cyclic nature of FSW process, plane strain filed converts to the shear planes. Fig. 7 shows a pattern for the transformation of plain strain to shear planes. 


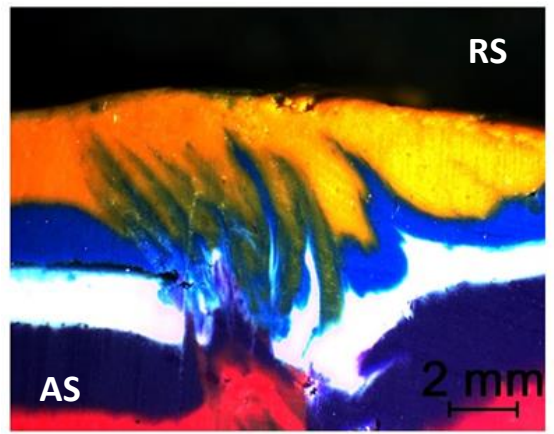

(a)

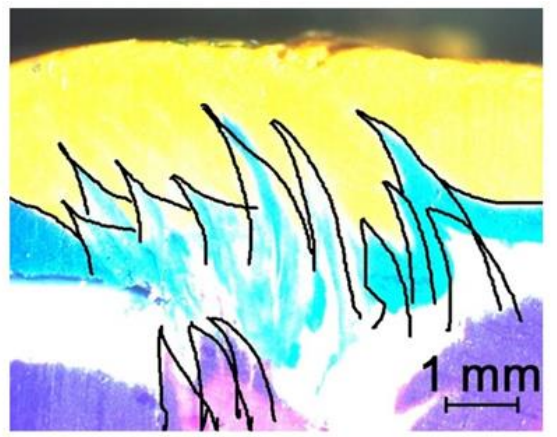

(b)

(c)

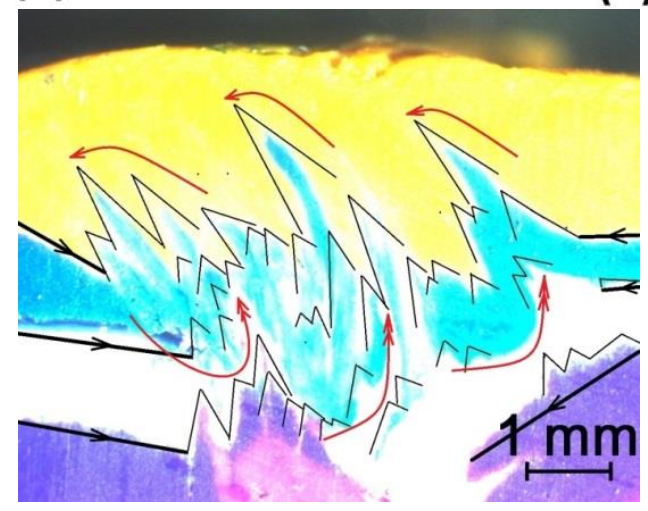

Fig. 5. Flow patterns representative of stress concentration; (a) dark-field exposure, (b) bright-field exposure, (c) proposed shear flow existing through the stress concentration region. Sample processed in $(\mathrm{V}=50 \mathrm{~mm} / \mathrm{min}$, $\omega=50 \mathrm{rpm}$ )

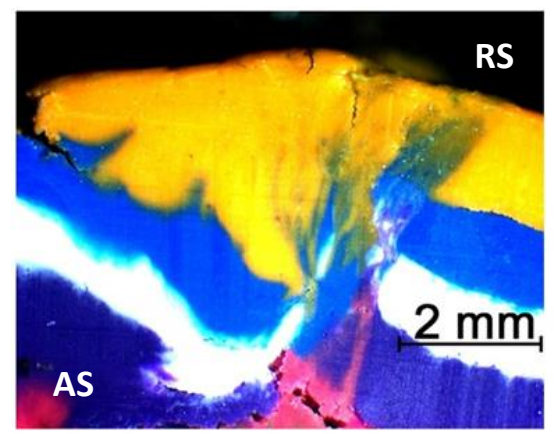

(a)

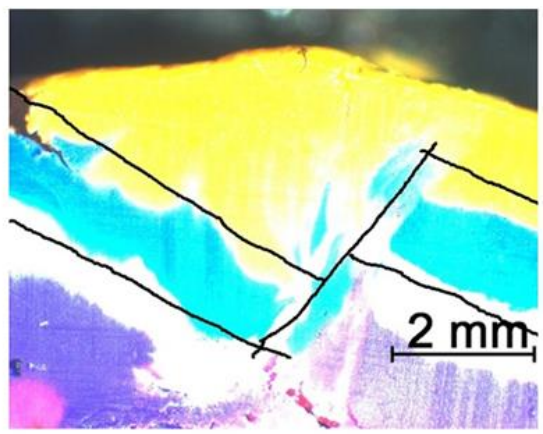

(b)

(c)

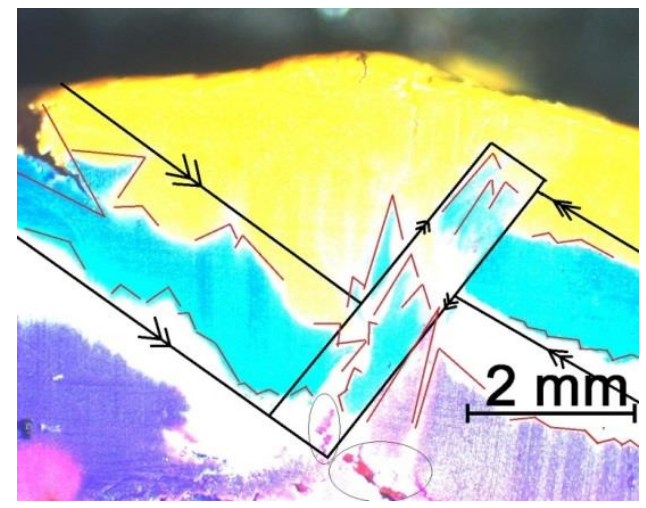

Fig. 6. Flow patterns representative of plane strain; (a) dark-field exposure, (b) bright-field exposure, (c) interaction of the flow layers between the orthogonal strained planes. Sample processed in $(\mathrm{V}=50 \mathrm{~mm} / \mathrm{min}$, $\omega=60 \mathrm{rpm}$ ) 


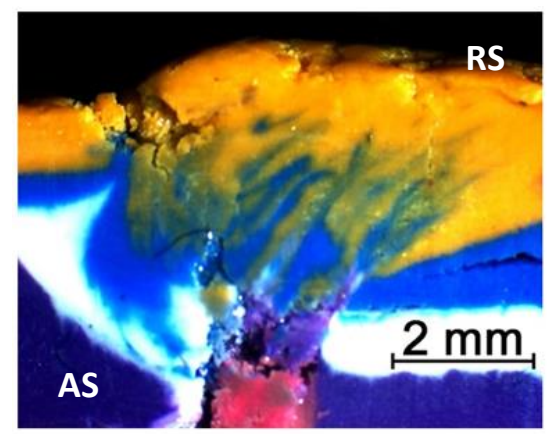

(a)

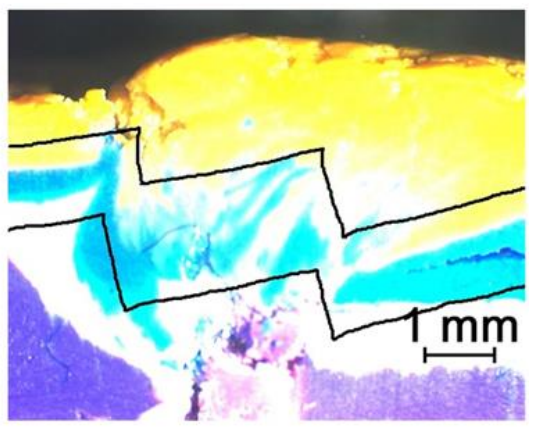

(b)

(c)

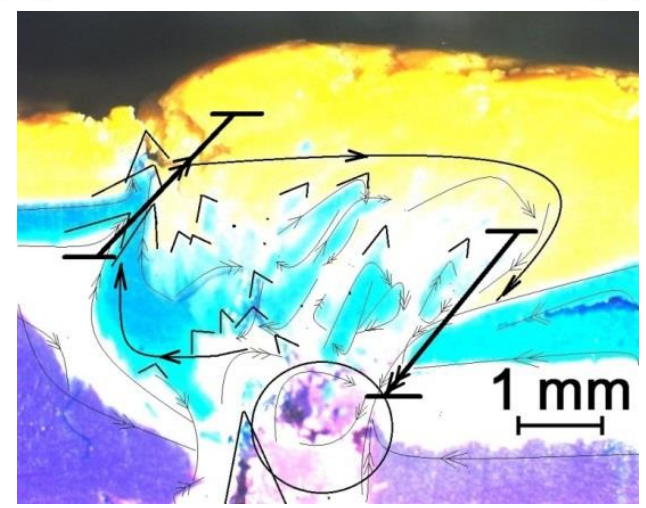

Fig. 7. Transformation of the flow patterns from plain strain to shear planes; (a) dark-field exposure, (b) brightfield exposure, (c) tangled shearing flow features with a swirling pattern through the shear planes. Sample processed in $(\mathrm{V}=50 \mathrm{~mm} / \mathrm{min}, \omega=75 \mathrm{rpm})$

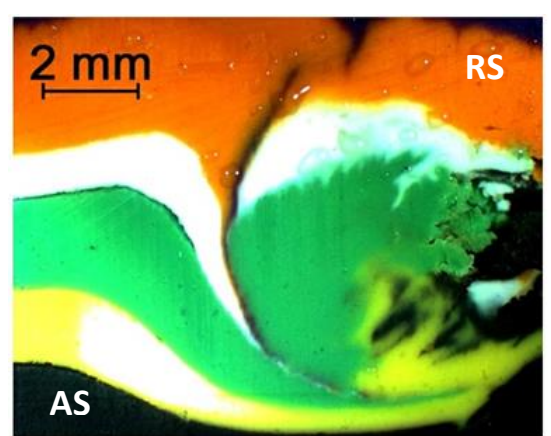

(a)

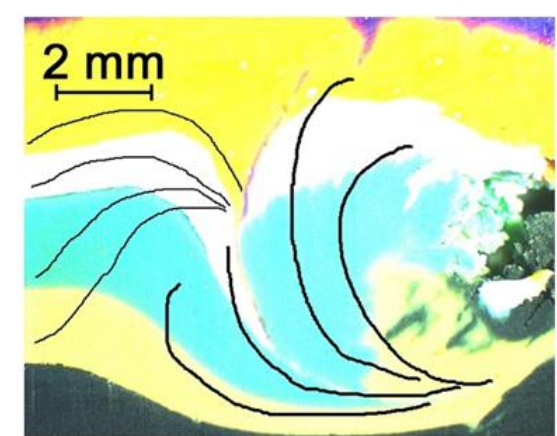

(b)

(c)

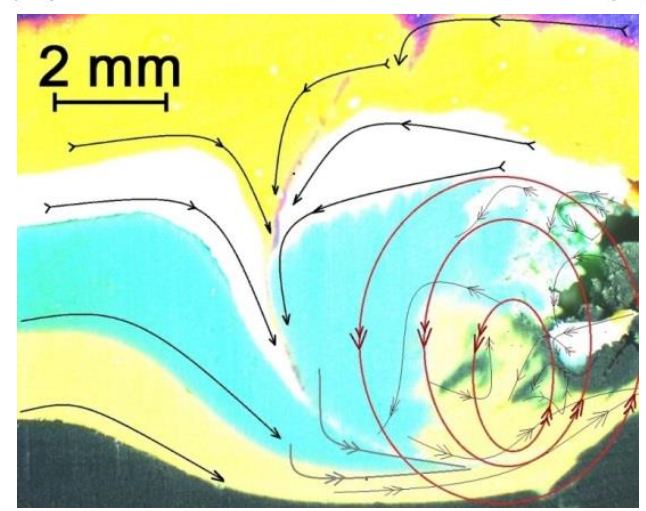

Fig. 8. Vortex patterns; destruction of shear planes in circulating flow; (a) dark-field exposure, (b) bright-field exposure, (c) turbulent shear flow behaviour in severe plastic deformation filed. Sample processed in $(\mathrm{V}=50 \mathrm{~mm} / \mathrm{min}, \omega=100 \mathrm{rpm})$ 
In BFSW process, the complex pin and shoulder generates a high rate of plastic deformation in the network. Rotation of the tool through the material shows it reduces the shear rate. Consequently, a rotating flow will be created in the structure. This vortex nature can damp all the stress-strain planes in a steady-state flow, (see Fig. 8). As the rotation of the tool is conducted in the interface of two plates, stirring also mixes the materials into a bonded structure which is called the nugget zone.

The flow patterns revealed in plasticine analogue visualization need to be validated by the aluminium weld samples. The etched weld cross-sections in Fig. 9 reveal the actual flow lines representative of the material transportation mechanism within the stirring zone. Fig. 9a shows the internal flow features within the transverse sections of the aluminium weld sample, processed at $(300 \mathrm{~mm} / \mathrm{min}$ and $400 \mathrm{RPM})$ welding speeds.

As it is observed in Fig. 9a, the shearing flow layers are representative of the severely distorted plastic flow on the border of the advancing side, as the effect of the simultaneous performance of the rotating pin and shoulder. Consequently, the clockwise rotating of the tool, causes a localized mixing of the plastic mass transferred towards the AS border. It is clear that the deposited plastic layers have a poor uniformity at the AS, in which the shearing effect of the tool disperses the mass flow in different directions during the deposition mechanism. This contributes to the formation of a discontinuity which is observed in Figures $9 \mathrm{~b}$ and $9 \mathrm{c}$.

Figures $9 \mathrm{~b}$ and $9 \mathrm{c}$ demonstrate the intensified shearing effect at higher speeds. The tangled flow patterns induced by the pin performance show a failure in flow dispersion whereby deteriorate the flow integrity during the mass refilling mechanism.

The insufficient flow mixing during mass transportation at the backside of the tool causes poor compaction between the flow layers. Consequently, by the formation of the downwards helical flow of mass, as the effect of the threads in the stirring zone, a tunnel void discontinuity forms at the lower layers of the separated flow lines.

In this situation, by the increase in welding speeds (Figure 9c), the elevated shearing effect within the plastic flow layers induces a vortex-like flow, which pushed the materials upwards and downwards and forms an open-hole tunnel void, located at the refilling position of the stirring zone towards the AS border.

The comparison between the flow patterns revealed in Fig. 9, indicate that the elevated rotational speed can significantly affect the stirring mechanism. By the intensified shearing effect around the rotating pin, the plastic flow undergoes an uncontrolled intermixing regime in which causes a subsequent adverse effect, interrupting the flow integrity during the refilling and mass deposition mechanism.

In general, the FSW welding mechanism suffers from insufficient material flow, during the stirring action. This results in the non-bonding discontinuity or void defect formation as the main weld flaw. The flow-based void defects can collapse the mass refilling mechanism for the deposition of the weld-line material, and subsequently, deteriorate the strength properties of the final weldment [26]. There are some other common defects during the FSW processes, which caused some difficulties to prevent them during the operational procedure. Some of these defects occur because of the improper selection of the welding parameters, leading to excessive or insufficient mass and heat flow. However, the formation mechanisms of these defects are not fully understood yet, and the body of the knowledge of the FSW process requires more scientific elucidation of the origins of defects within the weld structure. By understanding of the origins of the defects, and correct selection of the process parameters can obtain a sound weld with the optimum processing productivity [27, 28]. 

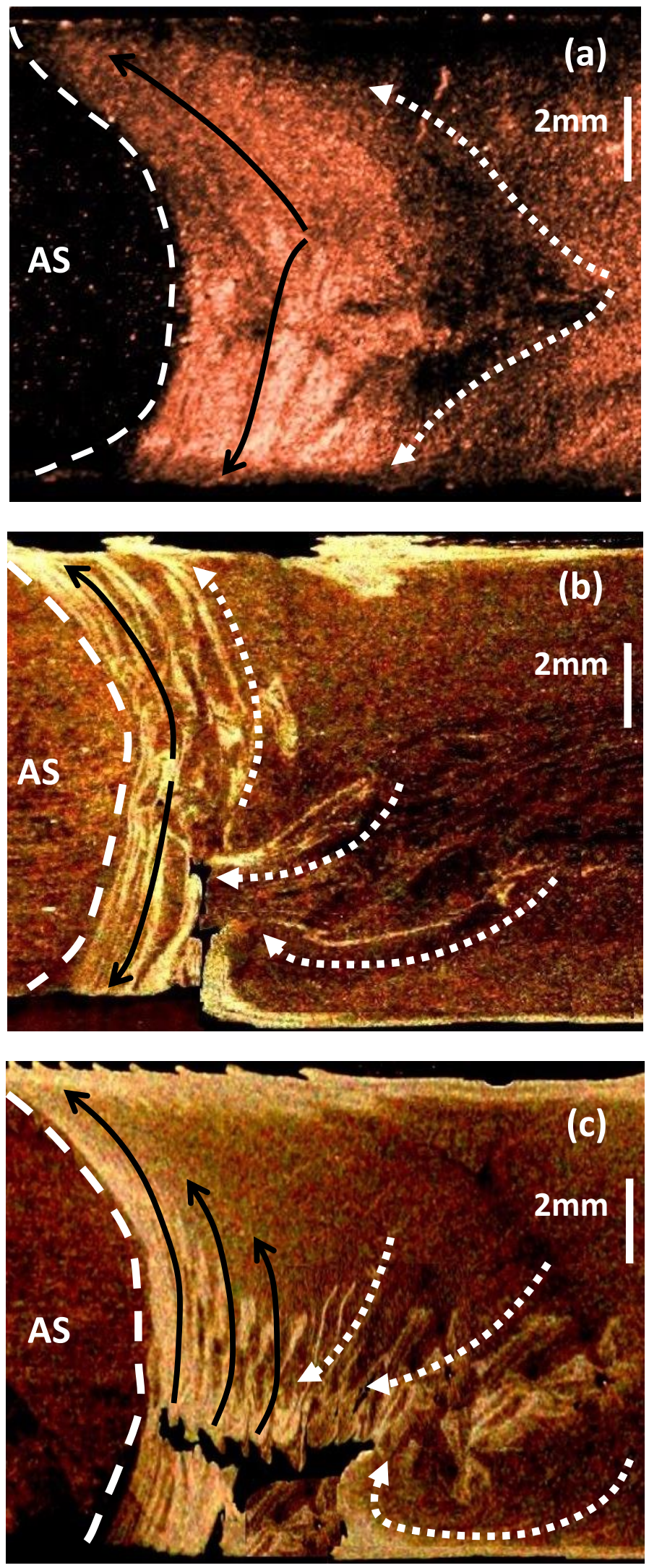

Fig. 9. Material flow patterns in aluminium cross-sections, welded in different speed sets; (a) $300 \mathrm{~mm} / \mathrm{min}$ and $400 \mathrm{RPM}$, (b) $300 \mathrm{~mm} / \mathrm{min}$ and $500 \mathrm{RPM}$, (c) $300 \mathrm{~mm} / \mathrm{min}$ and $600 \mathrm{RPM}$ 
As the characteristic of the bobbin-tool FSW, it seems to be a metal-forming process, as a combination of severe plastic deformation, forging and extrusion mechanisms, which finally results in a joining between two plates [29-31]. In fact, due to high mechanical loads, heat generation and material deformation and displacement, there are some complexities regarding the unknown-origin of the flow-based defects in relationship with the process parameters (e.g. longitudinal welding speed and spindle rotational speed), or geometrical features of the bobbin tool (size and geometry of pin and shoulder).

To achieve the optimum situation of the applicable process parameters, a solid theory is needed for a transition from trial-and-error to a science-based approach to extend the overall productivity [32,33]. To avoid the formation of the tunnel defect, a flow-based formation mechanism needs to be predicted according to the process variables.

The main aim of this study was to predict the based on the analogue model to elucidate. Based on the analogue modelling of the flow visualization a simplified physical model can summarize the general flow behaviour at the position of the tool-material interaction, during the stirring process. The fully-featured (threaded tri-flat pin) bobbin-tool can generate a specific inherent flow interaction around the tool.

By comparing the cross-sections of the plasticine analogue and aluminium welds, it was found that the discontinuity defect is commonly formed at the trailing edge of the tool, where the mass refilling mechanism is going to be complete there. In this regards, the mass transportation induced by the tool features and the welding speed can play the main role in plasticity of the stirring zone [34-37].

Based on the flow visualization identified in this work, the schematic of the mass flowlines (in plain-view), around the rotating tri-flat pin, is shown in Fig. 10. Fig. 10a shows that as the rotating pin enters into the substrate, the material undergoes a local discontinuity caused by the shearing effect of the stirring at the proximity of the tool, transporting the plasticized mass from the AS to the RS, by the clockwise rotation of the tool. The circulating of the stirred mass around the pin eventually leads to depositing at the trailing edge of the tool, where any inconsistency in flow integration can easily cause the inconsistency in flow regimes, and forming a mass deficit discontinuity between the refilled layers $[38,39]$.

It seems that during the helical flow path for transportation of the stirred mass around the rotating pin (from the leading edge to the trailing edge of the tool), the refilling inconsistency happens in form of the escape of a portion of the material at the backside of the tool (Figures $10 \mathrm{~b}$ and $10 \mathrm{c})$.

As the mass flow patterns around the pin is drawn in Figures $10 \mathrm{~b}$ and 10c, by sweeping the mass at the retreating side to the trailing edge of the tool, the possibility of the material deficit increases. The incomplete refilling of the stirred mass after circulation around the pin eventually emerge a discontinuity defect between the deposited mass layers at the backing edge of the pin.

By further advancement of the tool into the workpiece (Figures $10 \mathrm{~b}$ and 10c), the pin plasticizes more mass at the leading edge, transport it to the trailing edge of the pin by backwards squeezing. In this condition, the opposite direction of the linear and rotational movement of the pin creates an escaping force at the retreating side of the pin's edge with an intensifying slipping mode in mass transportation. This dispersive flow condition decreases the compression and integrity of the material layers, depositing at the trailing edge. 

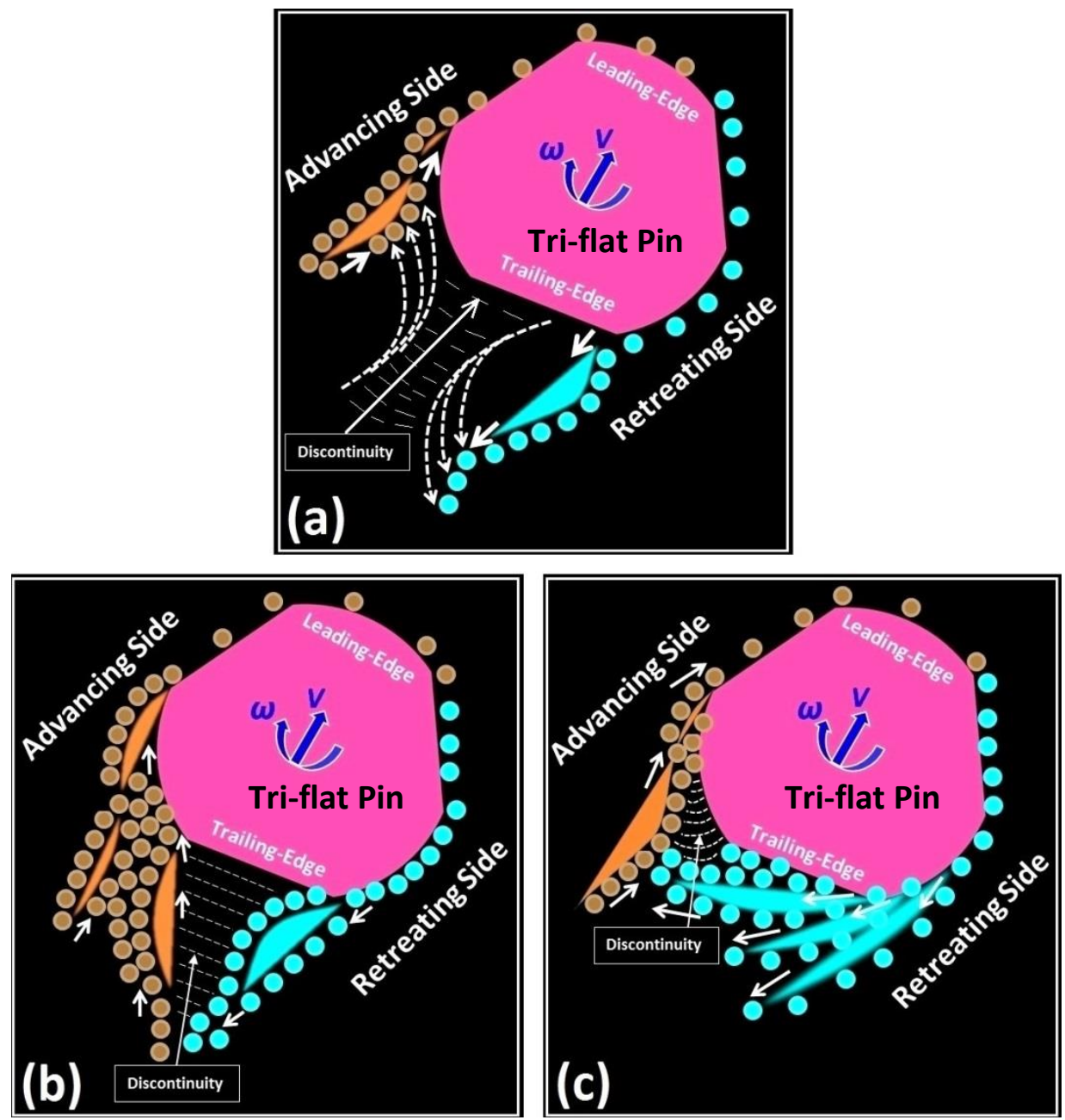

Fig. 10. The proposed physical model for the material flow patterns around the tri-flat pin; (a) entering of the rotating pin into the workpiece material, $(b, c)$ dispersive plastic flow mass with a discontinuity pattern formed at the trailing edge of the pin. (All schematics are presented at the top-view of the pin)

The discontinuity pattern observing at the trailing edge of the pin can eventually lead to emerging of the tunnel void (Fig. 10c), as the successive rotation of the pin and the RS-to-AS recirculating material flow intensifies the material deficit in forms a channel-shaped tunnel void as a flow-based defect.

By repeating this flow inconsistency behaviour during the stirring process, it seems that the lack of mixing and insufficient material refilling, at the backwards of the pin, are more effective in the evolution of the discontinuity defect.

The inconsistency between the linear and rotational speeds can interrupt the flow integrity during the refilling process. In this regards, the higher linear speed (V), gives less deposition time to the plastic mass flow at the backside of the tool [40]. Therefore, it causes the tearing of the weld-line bonding, resulting in a large discontinuity as the stirred mass has not enough time to be deposited at the back of the tool [41].

Similarly, in higher rotational speeds $(\omega)$, there is not enough displacement for the stirred mass around the tool to transport the plasticized mass with a suitable gap behind the tool. This interrupts the refilling mechanism, therefore the mass flow ejects from the stirring position in forms of the flash defect, spilt out from underneath the shoulder [42].

Therefore, the unsuitable ratio of welding speeds (linear to rotational speed), also ineffective tool geometry cause a flow partitioning instead of flow integrity during the stirring 
process and refilling mechanism of the plastic mass. The interruption in flow integrity eventually emerges a discontinuity between the deposited mass layers in form of the tunnel void defect, situated near the position of the AS border of the SZ, where the failure of the refilling mechanism happens.

\section{SUMMARY}

This research illustrates an original contribution by introducing analogue modelling of the BFSW process to visualize plastic deformation and joining of material. The method is applicable to explain material flow trajectories during welding. Failure of flow regimes in each step of the joining process can cause defects in the structure. A successful flow regime can result in a sustainable joint through the weld line. The similarity between the flow pattern of the provided aluminium samples and the plasticine analogue can validate the accuracy of the flow model presented in this work. Also it is suggested that a flow pattern for the formation of the nugget zone or stirring area as a feature for FSW welds. The failure of the flow appears during the refilling mechanism results in the formation of the tunnel void discontinuity within the stirring zone. The proposed flow-based model shows explained different flow dispersion which interrupting the flow integrity at the backside of the tool.

\section{ORCID iD:}

Abbas Tamadon $\quad$ https://orcid.org/0000-0001-7367-4207

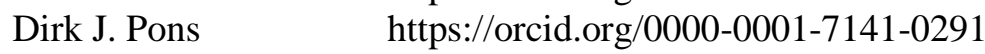

Don Clucas ～https://orcid.org/0000-0002-6724-3037

\section{REFERENCES}

1. Tashkandi MA, Al-Jarrah JA, Ibrahim M. Increasing of the Mechanical Properties of Friction Stir Welded Joints of 6061 Aluminum Alloy by Introducing Alumina Particles. Advances in Materials Science. 2017, 17(2), 29-40.

2. Thomas, W.; Nicholas, E.; Needham, J.; Murch, M.; Temple-Smith, P.; Dawes, C. Friction stir butt welding, international patent application no. PCT/GB92 Patent application 1991.

3. Thomas, W.; Wiesner, C.; Marks, D.; Staines, D. Conventional and bobbin friction stir welding of $12 \%$ chromium alloy steel using composite refractory tool materials. Science and Technology of Welding and Joining 2009, 14, 247-253.

4. Threadgill, P.; Leonard, A.; Shercliff, H.; Withers, P. Friction stir welding of aluminium alloys. International Materials Reviews 2009, 54, 49-93.

5. Fuse, K.; Badheka, V. Bobbin tool friction stir welding: A review. Science and Technology of Welding and Joining 2019, 24, 277-304.

6. Goetze, P.; Kopyściański, M.; Hamilton, C.; Dymek, S. Comparison of dissimilar aluminum alloys joined by friction stir welding with conventional and bobbin tools. In Friction stir welding and processing X, Springer: 2019, 3-12.

7. Iwaszko, J.; Kudła, K. Effect of friction stir processing (FSP) on microstructure and hardness of almg10/sic composite. Bulletin of the Polish Academy of Sciences. Technical Sciences 2019, 67, 185-192. 
8. Yang, C.; Ni, D.; Xue, P.; Xiao, B.; Wang, W.; Wang, K.; Ma, Z. A comparative research on bobbin tool and conventional friction stir welding of al-mg-si alloy plates. Materials Characterization 2018, 145, 20-28.

9. Xu, W.; Luo, Y.; Fu, M. Microstructure evolution in the conventional single side and bobbin tool friction stir welding of thick rolled 7085-t7452 aluminum alloy. Materials Characterization 2018, $138,48-55$.

10. Kalemba-Rec, I.; Kopyściański, M.; Miara, D.; Krasnowski, K. Effect of process parameters on mechanical properties of friction stir welded dissimilar 7075-T651 and 5083-H111 aluminum alloys. The International Journal of Advanced Manufacturing Technology 2018, 97, 2767-2779.

11. Tamadon, A.; Pons, D.J.; Clucas, D. Structural anatomy of tunnel void defect in bobbin friction stir welding, elucidated by the analogue modelling. Applied System Innovation 2020, 3, 2.

12. Tamadon, A.; Pons, D.J.; Clucas, D.; Sued, K. Internal material flow layers in AA6082-T6 buttjoints during bobbin friction stir welding. Metals 2019, 9, 1059.

13. Kluz, R.; Kubit, A.; Trzepiecinski, T.; Faes, K.; Bochnowski, W. A weighting grade-based optimization method for determining refill friction stir spot welding process parameters. Journal of Materials Engineering and Performance 2019, 28, 6471-6482.

14. Tamadon, A.; Pons, D.; Sued, M.; Clucas, D.; Wong, E. Preparation of plasticine material for analogue modelling, Proceedings of the International Conference on Innovative Design and Manufacturing (ICIDM2016), Auckland, New Zealand, 24-26 January 2016, 2016; Auckland, New Zealand.

15. Tamadon, A.; Pons, D.; Sued, M.; Clucas, D.; Wong, E. Analogue modelling of bobbin tool friction stir welding, Proceedings of the International Conference on Innovative Design and Manufacturing (ICIDM2016), Auckland, New Zealand, 24-26 January 2016, 2016; Auckland, New Zealand.

16. Tamadon, A.; Pons, D.; Sued, K.; Clucas, D. Formation mechanisms for entry and exit defects in bobbin friction stir welding. Metals 2018, 8, 33.

17. Sued, M.; Tamadon, A.; Pons, D. Material flow visualization in bobbin friction stir welding by analogue model. Proceedings of Mechanical Engineering Research Day 2017, 2017, 1-2.

18. Tamadon, A.; Pons, D.; Sued, K.; Clucas, D. Development of metallographic etchants for the microstructure evolution of A6082-T6 BFSW welds. Metals 2017, 7, 423.

19. Tamadon, A.; Pons, D.; Sued, K.; Clucas, D. Thermomechanical grain refinement in AA6082-T6 thin plates under bobbin friction stir welding. Metals 2018, 8, 375.

20. Liu, X.; Wu, C.; Padhy, G.K. Characterization of plastic deformation and material flow in ultrasonic vibration enhanced friction stir welding. Scripta Materialia 2015, 102, 95-98.

21. Tamadon, A.; Pons, D.J.; Clucas, D.; Sued, K. Texture evolution in AA6082-T6 BFSW welds: Optical microscopy and EBSD characterisation. Materials 2019, 12, 3215.

22. Tamadon, A.; Pons, D.J.; Clucas, D. AFM characterization of stir-induced micro-flow features within the AA6082-T6 BFSW welds. Technologies 2019, 7, 80.

23. Padhy, G.; Wu, C.; Gao, S. Friction stir based welding and processing technologies-processes, parameters, microstructures and applications: A review. Journal of Materials Science \& Technology 2018, 34, 1-38.

24. Sued, M.; Pons, D.; Lavroff, J.; Wong, E.-H. Design features for bobbin friction stir welding tools: Development of a conceptual model linking the underlying physics to the production process. Materials \& Design 2014, 54, 632-643.

25. Rhodes, C.; Mahoney, M.; Bingel, W.; Spurling, R.; Bampton, C. Effects of friction stir welding on microstructure of 7075 aluminum. Scripta Materialia 1997, 36, 69-75. 
26. Colligan, K. Material flow behavior during friction welding of aluminum. Welding Journal 1999, 75, 229-237.

27. Dialami, N.; Cervera, M.; Chiumenti, M. Effect of the tool tilt angle on the heat generation and the material flow in friction stir welding. Metals 2019, 9, 28.

28. Dialami, N.; Chiumenti, M.; Cervera, M.; Agelet de Saracibar, C.; Ponthot, J.P. Material flow visualization in friction stir welding via particle tracing. International Journal of Material Forming 2015, 8, 167-181.

29. He, X.C. Computational investigation of mechanical behaviour of FSW joints, Applied Mechanics and Materials, 2013, 389, 260-266.

30. Hilgert, J.; Dos Santos, J.; Huber, N. Shear layer modelling for bobbin tool friction stir welding. Science and Technology of Welding and Joining 2012, 17, 454-459.

31. Hilgert, J.; Hütsch, L.L.; dos Santos, J.; Huber, N. In Material flow around a bobbin tool for friction stir welding, Excerpt from the Proceedings of the COMSOL Conference, 2010.

32. Gadakh, V.S.; Kumar, A.; Vikhe Patil, G.J. Analytical modeling of the friction stir welding process using different pin profiles. Welding Journal 2015, 94, 115-124.

33. He, X.; Gu, F.; Ball, A. A review of numerical analysis of friction stir welding. Progress in Materials Science 2014, 65, 1-66.

34. Khan, N.Z.; Siddiquee, A.N.; Khan, Z.A.; Shihab, S.K. Investigations on tunneling and kissing bond defects in fsw joints for dissimilar aluminum alloys. Journal of Alloys and Compounds 2015, 648, 360-367.

35. Kim, S.-D.; Yoon, J.-Y.; Na, S.-J. A study on the characteristics of FSW tool shapes based on CFD analysis. Welding in the World 2017, 61, 915-926.

36. Liechty, B.; Webb, B. Modeling the frictional boundary condition in friction stir welding. International Journal of Machine Tools and Manufacture 2008, 48, 1474-1485.

37. Moradi, M.M.; Aval, H.J.; Jamaati, R. Effect of tool pin geometry and weld pass number on microstructural, natural aging and mechanical behaviour of sic-incorporated dissimilar frictionstir-welded aluminium alloys. Sādhanā 2019, 44, 9.

38. Patel, V.; Li, W.; Vairis, A.; Badheka, V. Recent development in friction stir processing as a solid-state grain refinement technique: Microstructural evolution and property enhancement. Critical Reviews in Solid State and Materials Sciences 2019, 44, 378-426.

39. Tamadon, A.; Pons, D.J.; Clucas, D. Flow-based anatomy of bobbin friction-stirred weld; AA6082-T6 aluminium plate and analogue plasticine model. Applied Mechanics 2020, 1, 3-19.

40. Tamadon, A.; Pons, D.J.; Clucas, D. Microstructural study on thermomechanical behaviour of 6082-T6 aluminium BFSW weld plates. In Materials@UC 2018 Conference, Christchurch, New Zealand, 2018.

41. Tamadon, A.; Pons, D.J.; Clucas, D. Thermomechanical performance of bobbin tool design as an innovative variant for friction stir welding. In Manufacturing and Design Conference (MaD 2019) Auckland, New Zealand, 2019.

42. Tamadon, A. Characterization of flow-based bobbin friction stir welding process. Ph.D. Thesis, University of Canterbury, Christchurch, New Zealand, 2019. 